\title{
A model based on endoscopic morphology of submucosal esophageal squamous cell carcinoma for determining risk of metastasis on lymph nodes
}

\author{
Lingdun Zhuge ${ }^{1,2 \#}$, Shengfei Wang ${ }^{1,2 \#}$, Juntao Xie ${ }^{1,2}$, Binhao Huang ${ }^{1,2}$, Difan Zheng ${ }^{1,2}$, Shanbo Zheng ${ }^{1,2}$, \\ Hengyu Mao ${ }^{1,2}$, Arjun Pennathur ${ }^{3}$, Manuel Villa Sanchez ${ }^{3}$, James D. Luketich ${ }^{3}$, Jiaqing Xiang ${ }^{1,2}$, \\ Haiquan Chen ${ }^{1,2}$, Jie Zhang ${ }^{1,2,3}$ \\ ${ }^{1}$ Department of Thoracic Surgery, Fudan University Shanghai Cancer Center, Shanghai 200032, China; ${ }^{2}$ Department of Oncology, Shanghai \\ Medical College, Fudan University, Shanghai 200032, China; ${ }^{3}$ Department of Cardiothoracic Surgery, University of Pittsburgh School of Medicine, \\ Pittsburgh, PA 15213, USA \\ Contributions: (I) Conception and design: J Zhang, L Zhuge, S Wang; (II) Administrative support: J Zhang; (III) Provision of study materials or \\ patients: J Zhang, H Chen, J Xiang; (IV) Collection and assembly of data: L Zhuge, S Wang, B Huang; (V) Data analysis and interpretation: J Zhang, \\ L Zhuge, S Wang, J Xie, B Huang, D Zheng, S Zheng, H Mao, I Sarkaria, M Sanchez, J Luketich; (VI) Manuscript writing: All authors; (VII) Final \\ approval of manuscript: All authors. \\ "These authors contributed equally to this work. \\ Correspondence to: Jie Zhang, MD, PhD. Department of Thoracic Surgery, Fudan University Shanghai Cancer Center, 270 Dong-An Road, Shanghai \\ 200032, China. Email: zhangj19@upmc.edu.
}

Background: It is important to identify patients with esophageal squamous cell carcinoma (ESCC) in T1b stage that are the least likely to metastasize on the lymph nodes, to undergo endoscopic resection, especially for the patients unfit for esophagectomy. The relationship between endoscopic morphology and frequency of nodal metastasis has never been well studied. The aims of the study were to investigate the predictive value of endoscopic morphology for lymphatic metastasis, and to develop a risk stratification model in submucosal (T1b) ESCC.

Methods: Pathologic variables of patients with T1b ESCC who underwent esophagectomy from 2006 through 2016 were collected and divided into training sets (patients between 2006 and 2011) and validation sets (patients between 2012 and 2016). The endoscopic morphology of the tumor was determined by analyzing endoscopic reports according to the Paris classification. The correlation between the clinicopathological factors and nodal metastasis was examined. A prediction model was developed to estimate the risk of metastasis using these predictors.

Results: A total of 175 patients were included in this study. A tumor with an endoscopic shape of flat type (0-II type as Paris classification was defined) was significantly related to lower risk of lymphatic metastasis with the frequency of $15.5 \%$ (OR: 3.049, 95\% CI: 1.363-6.819, $\mathrm{P}=0.005$ ). The combination of endoscopic morphology with other pathologic characteristics including lymphovascular invasion, length of tumor, depth of tumor invasion into submucosa, and tumor differentiation improved the predictive value of the nodal metastasis. The risk stratification model was developed with a C-index of 0.726 (95\% CI: 0.702-0.751), which identified a low risk subgroup with a lymph node rate of $7.2 \%$.

Conclusions: Our results suggest that when a tumor is in flat shape (0-II type) it is related to a less lymphatic metastasis, and the combination of the endoscopic morphology with the other four pathologic variables can yield a more robust approach to predict the risk of lymphatic metastasis in submucosal ESCC.

Keywords: Esophageal carcinoma; submucosal; flat type; lymph node metastases 
Submitted Aug 08, 2018. Accepted for publication Nov 15, 2018.

doi: $10.21037 /$ jtd.2018.11.77

View this article at: http://dx.doi.org/10.21037/jtd.2018.11.77

\section{Introduction}

Superficial esophageal cancer including intraepithelial, mucosal and submucosal cancers, implies a better prognosis owing to an early tumor stage. The recent increasing trend towards endoscopic resection (ER) of superficial esophageal squamous cell carcinoma (ESCC) leads to a lower morbidity and mortality in comparison with a traditional esophagectomy $(1,2)$. However, because of the limitation of endoscopic treatment that lymphadenectomy is unavailable during the procedure and no lymph node is removed, the potential risk of nodal metastasis cannot be fully excluded preoperatively, which is significantly related to poorer survival.

In $\mathrm{T} 1$ cancer, compared to the only $5 \%$ of patients with a nodal metastasis in a T1a tumor (intramucosal cancer), the frequency increases to $24-33 \%$ when the tumor invades into the submucosal layer $(3,4)$. Although there are several predictors for nodal metastasis which have been identified by previous studies, such as lymphovascular invasion (LVI), tumor invasion to deepest one-third layer of submucosa (sm3), and poorer differentiation (5-7), it has been inadequate in determining a subgroup with a low risk of nodal metastasis in submucosal cancers using a single factor. Thus, tumors in the superficial one-third layer of submucosa (sm1) are considered as relative indication for ER, and the appropriateness of application of ER to malignant lesions deeper than sm1 still remains controversial $(8,9)$.

In patients with T1b (submucosal cancer), ESCC patients who are unfit for surgical resection, such as the elderly or those who reject esophagectomy, therapeutic strategies have to be compromised, and ER becomes a more suitable clinical choice $(10,11)$. For those patients, the nodal status is critical for further clinical decisions subsequent to ER regarding whether or not to perform radiation therapy or have an intensive follow-up.

Endoscopic appearance of superficial tumor based on the Paris endoscopic classification (12) was divided into 3 types as protruding (0-I type), excavated (0-III type) and flat type (0-II type). Previous studies suggested endoscopic appearance of the tumor was related to the depth of the tumor invasion and lymph node status in several cancer types $(13,14)$. However, the predictive value of the tumor shape for a nodal metastasis, especially in submucosal ESCC, has never been well studied (15). Unlike pathological factors, it is unproblematic to get the information for tumor shape, before surgical resection, and it is more convenient to assess the lymph node status preoperatively according to the macroscopic appearance.

The aims of this study were to determine the correlation between endoscopic types and nodal metastasis, and to develop a model that incorporated both endoscopic gross morphology and other pathologic risk factors for prediction of lymph node metastasis in T1b ESCC.

\section{Methods}

\section{Patient/case selection}

Patients who underwent an esophagectomy procedure from 2006 to 2016 in the Department of Thoracic Surgery, Fudan University Shanghai Cancer Center were collected. All the cases diagnosed as primary ESCC at T1b stage and confirmed as sm1-sm 3 tumor by postoperative pathology were selected. The electronic medical records and physical charts of the selected patients were reviewed for patient and treatment- variables. The representative slides were reviewed by 2 independent pathologists in order to score the following pathologic features of the tumor: depth of invasion, tumor differentiation, LVI and tumor size. The original reports of postoperative pathology were reviewed after all these procedures to determine nodal metastatic status.

The patient inclusion criteria for our retrospective study were as follows: (I) thoracic submucosal ESCC at pathological sm1-sm3 stage; (II) radical lymphadenectomy through a thoracotomy; (III) 12 or more removed lymph nodes (16); (IV) no chemotherapy or radiotherapy before surgery; and (V) specimens available for repeat review.

In order to assess the predictive value of multivariable model, the enrolled patients were divided into 2 independent sets. Patients who underwent a surgical resection from 2006 to 2011 were included in the training set, and the remaining between 2012 and 2016 were taken into the validation set. 
Table 1 Patient clinical characteristics

\begin{tabular}{|c|c|}
\hline Characteristic & Patients number (\%) \\
\hline \multicolumn{2}{|l|}{ Gender } \\
\hline Female & $43(24.6)$ \\
\hline Male & $132(75.4)$ \\
\hline \multicolumn{2}{|l|}{ Age (y) } \\
\hline$\leq 60$ & $77(44.0)$ \\
\hline$>60$ & $98(56.0)$ \\
\hline \multicolumn{2}{|l|}{ Type } \\
\hline Flat & $58(33.1)$ \\
\hline Non-flat & $117(66.9)$ \\
\hline \multicolumn{2}{|c|}{ Lymphovascular invasion } \\
\hline Negative & $143(81.7)$ \\
\hline Positive & $32(18.3)$ \\
\hline \multicolumn{2}{|l|}{ Differentiation } \\
\hline Well & $41(23.4)$ \\
\hline Moderate & $88(50.3)$ \\
\hline Poor & $46(26.3)$ \\
\hline \multicolumn{2}{|l|}{ Tumor Length (cm) } \\
\hline$<3$ & $123(70.3)$ \\
\hline$\geq 3$ & $52(29.7)$ \\
\hline \multicolumn{2}{|l|}{ Tumor depth } \\
\hline SM1 & $38(21.7)$ \\
\hline SM2 & $75(42.9)$ \\
\hline SM3 & $62(35.4)$ \\
\hline \multicolumn{2}{|c|}{ Pre-operative clinical N stage } \\
\hline Negative (cNO) & $142(81.1)$ \\
\hline Positive (cN1) & $31(17.7)$ \\
\hline Unknown (cNx) & $2(1.1)$ \\
\hline \multicolumn{2}{|l|}{ LNM } \\
\hline Negative & $124(70.9)$ \\
\hline Positive & $51(29.1)$ \\
\hline
\end{tabular}

LNM, lymph node metastasis.

\section{Definition of endoscopic classification}

According to the Paris endoscopic classification (12), which classifies based on the gross morphological appearance of the superficial esophageal lesions, all the selected patients were divided into 3 types: protruding type (0-I), flat type (0-II) and excavated type (0-III). Due to the similar rate of lymph node metastasis in this study $(\mathrm{P}=0.787)$, protruding $(36.5 \%)$ and excavated type $(33.3 \%)$ were combined as one type named the non-flat type.

\section{Statistical analysis}

Categorical variables were presented as categories and percentages. Either the Chi-square test or Fisher's exact test was used to assess the significance of the differences in the univariate analysis. All factors found to be statistically significant were examined using logistic regression in the multivariable analysis to identify independent risk factors. Relevant clinical and pathologic factors were all included in a multivariable model designed using logistic regression to predict the risk of nodal metastasis. The internal validation of the nomogram was performed by bootstraps with 1,000 resamples. The external validation was evaluated with the validation set. Its predictive accuracy and discriminatory capacity were determined using the concordance index (C-index) and the calibration curve (17). Statistical analyses were performed with SPSS, version 20.0 (IBM, Armonk, NY), and R software packages, version 3.1.2 (The R Foundation for Statistical Computing, http://www.r-project.org/). A two-sided $\mathrm{p}$ value of less than 0.05 was considered to be statistically significant for all reports.

\section{Results}

\section{Clinical and pathological characteristics}

In total, 175 patients with ESCC in a pathological sm1-sm3 stage were included in this study. The majority were males and the median age was 61 years (interquartile range, 4477 years). Fifty-one patients (29.1\%) had at least one lymph node metastasis according to a postoperative pathology report (Table 1).

\section{Association between clinicopathological characteristics and nodal metastasis}

Endoscopic flat-type (0-II type) were found in 58 patients (33.1\%) with submucosal invasion (pT1b). Among these patients, $9(15.5 \%)$ were identified as having a nodal metastasis by postoperative pathology compared to $35.9 \%$ patients with non-flat type (0-I or 0-III type) ESCC (OR: 
Table 2 Frequency of lymph node metastases according to tumor characteristics

\begin{tabular}{|c|c|c|c|c|}
\hline Type of analysis & Positive No. (\%) & OR & $95 \% \mathrm{Cl}$ & $P$ value \\
\hline \multicolumn{5}{|l|}{ Univariate } \\
\hline Gender & & & $0.505-2.326$ & 0.837 \\
\hline Female & $12(27.9)$ & 1.000 & & \\
\hline Male & 39 (29.5) & 1.083 & & \\
\hline Age (y) & & & $0.390-1.446$ & 0.391 \\
\hline$\geq 60$ & $26(26.5)$ & 0.751 & & \\
\hline Type & & & $1.363-6.819$ & 0.005 \\
\hline Flat & $9(15.5)$ & 1.000 & & \\
\hline Non-flat & $42(35.9)$ & 3.049 & & \\
\hline Differentiation & & & $0.697-2.943$ & 0.327 \\
\hline Well/moderate & $35(27.1)$ & 1.000 & & \\
\hline Poor & $16(34.8)$ & 1.432 & & \\
\hline Tumor length (cm) & & & $1.053-4.187$ & 0.033 \\
\hline$<3$ & $30(24.4)$ & 1.000 & & \\
\hline$\geq 3$ & $21(40.4)$ & 2.100 & & \\
\hline Tumor depth & & & $1.457-5.617$ & 0.001 \\
\hline SM1/SM2 & $24(21.2)$ & 1.000 & & \\
\hline Tumor depth & - & 1.960 & $0.942-4.081$ & 0.072 \\
\hline
\end{tabular}

3.049, 95\% CI: $1.363-6.819, \mathrm{P}=0.005)$. Additionally, the odds of a nodal metastasis were significantly increased in the presence of LVI, tumor length larger than $3.0 \mathrm{~cm}$, and deep submucosal invasion (sm3). A tumor with a poor differentiation value was more likely to have a higher risk of developing a lymph node metastasis, but the P-value was not statistically significant (Table 2).

\section{Multivariable analysis of association between clinicopathological characteristics and nodal metastasis}

All four risk factors for a nodal metastasis were confirmed by univariate analysis including endoscopic type, LVI, tumor length, and tumor depth, which were taken into a multivariable logistic regression model to identify the independent predictors. The results demonstrated that an endoscopic non-flat type associated with 2.622 -fold (95\% CI: $1.120-6.329, \mathrm{P}=0.027)$ increased the risk of lymph node metastasis, and LVI (OR: 4.567, 95\% CI: 1.933-10.791, $\mathrm{P}=0.001)$ was identified to be independent predictors for lymph node metastasis (Table 2).

\section{The effect of endoscopic type on the risk of lymph node metastasis in combination with other risk factors}

Due to the low frequency of a nodal metastasis, further 
A

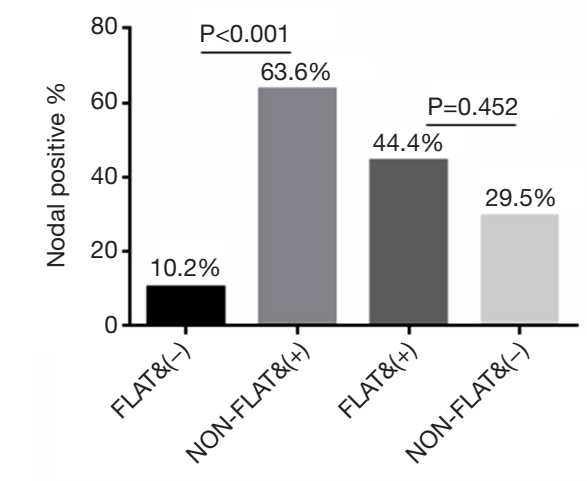

C

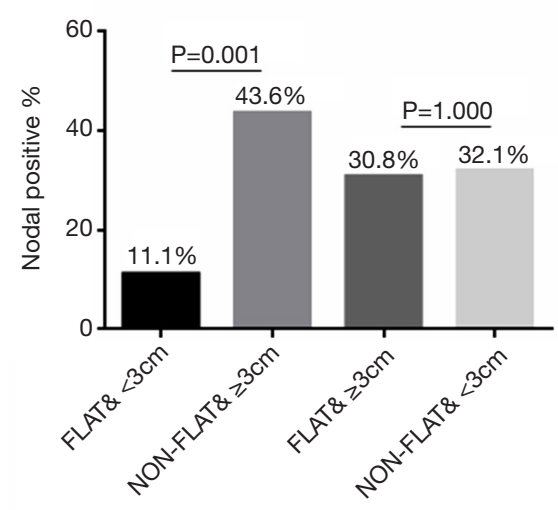

B Endoscopic Type and Depth

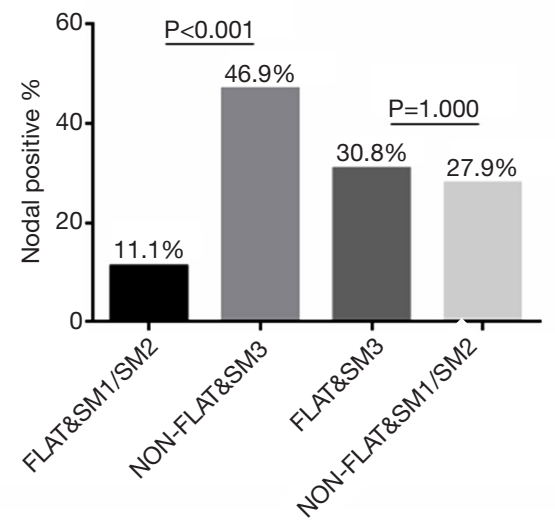

D Endoscopic Type and Differentiation

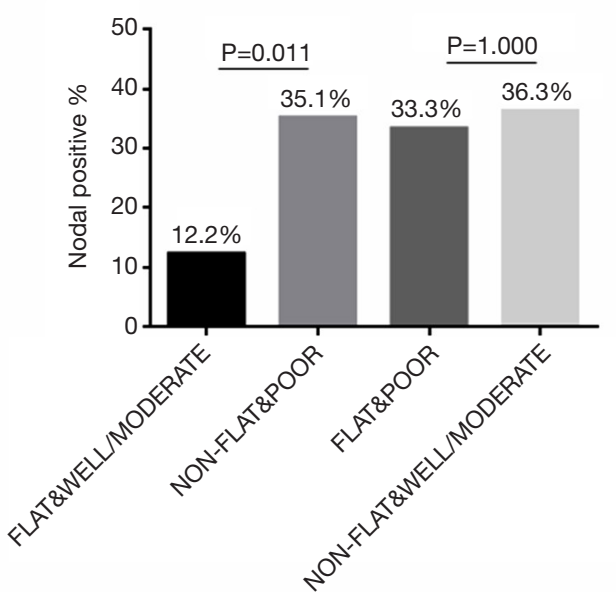

Figure 1 Bar graphs illustrating the prevalence of node metastasis in cases classified based on the presence of tumor endoscopic morphology and (A) lymphovascular invasion; (B) depth of submucosal invasion; (C) tumor length; (D) tumor differentiation.

analysis using combinations of risk factors was performed in this subgroup in order to illustrate how the endoscopic type modified the odds of a lymph node metastasis. When cases were grouped based on endoscopic type and LVI, only 5 out of $49(10.2 \%)$ of flat-type (0-II type) tumors without LVI were node positive compared with 14 out of $22(63.6 \%)$ of non-flat type $(0-\mathrm{I}$ or $0-\mathrm{III}$ type) with LVI $(\mathrm{P}<0.001)$. Similarly, the combination of endoscopic classifications with the depth of tumor invasion significantly influenced the frequency of a lymph node metastasis. Nodal metastasis occurred in 5 out of $45(11.1 \%)$ patients with a flat-type tumor with superficial submucosal invasion, and 23 out of $49(46.9 \%)$ patients of non-flat type with deep submucosal invasion $(\mathrm{P}<0.001)$. Additionally, flat type tumors with a size smaller than $3 \mathrm{~cm}$ or well to moderate differentiation were relatively lower risks (Figure 1).

\section{A model predicting nodal metastasis in submucosal ESCC}

A logistic regression model based on endoscopic morphology and four other pathological factors was designed to predict the risk of a nodal metastasis in submucosal ESCC using the training set data (104 patients). For the convenience of the clinical practice, the model was presented as a nomogram that graphically showed the multivariate effects of the risk factors (Figure 2). The C-index of the model was 0.726 (95\% CI: $0.702-0.751)$. In order to assess the accuracy of the predictive value, the model was examined with validation 


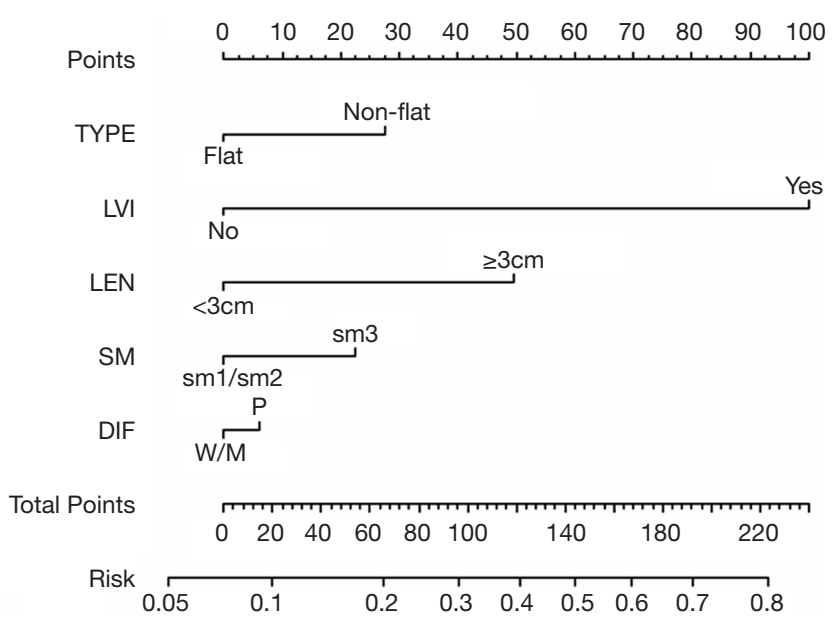

Figure 2 Nomogram predicting risk of lymph node metastases in submucosal cancers. Nomogram instructions: to obtain the nomogram-predicted risk of lymph node metastases, locate the patient values on each axis. Draw a vertical line to the points axis to determine how many points are attributable to each variable. Sum the points for all the variables. Locate the sum on the total points line to assess the patient's predicted risk of lymph node metastases. LVI, lymphovascular invasion; LEN, length of tumor; SM, depth of tumor invasion into submucosa; DIF, tumor differentiation; $W$, well; $M$, moderate; $\mathrm{P}$, poor.

set data (71 patients). The results demonstrated the model yielded a satisfied C-index of 0.748 (95\% CI: $0.633-0.863$ ) for predicting nodal metastasis. The calibration curve for the probability of metastasis to lymph node showed consistency between the prediction of nomogram and actual observations in the two sets (Figure S1).

The model identified a "low risk" subgroup with a relatively low nodal metastasis rate of $7.3 \%$, which was defined as having a tumor in an endoscopic flat shape (0-II type) with no LVI, sm1 or sm2 invasion, well or moderate differentiation and tumor length less than $3 \mathrm{~cm}$.

\section{Discussion}

The past decade has witnessed the dramatic change for the management of esophageal carcinoma, owing to the widespread usage of novel endoscopic technology. The trend towards endoscopic resection in patients with superficial esophageal carcinoma has significantly reduced postoperative complications in comparison to esophagectomy (18) and has improved the quality of life by allowing patients keep their esophagus. Unlike loco- regionally advanced cancer where metastasis happens commonly, T1 esophageal cancer is a more appropriate indicator for endoscopic treatment because it is typically curable with just a resection of the lesion alone. However, the risk of lymphatic metastasis is a major concern for the candidates of endoscopic resection, since lymphadenectomy is not available to remove the potentially involved lymph nodes. Thus, it is important and necessary to have a clear understanding of the risk of nodal metastasis preoperatively.

Predictive models for nodal status seem to be more crucial in the patients with an early stage cancer, but unsuitable for esophagectomy or patients unwilling to receive it, and for these patients ER becomes a more appropriate treatment $(10,11)$. The further therapeutic strategies such as radiotherapy could be applied for those with lymph node metastases, in order to achieve more favorable prognosis. Thus, precise prediction of lymph node status is necessary.

Based on a consensus in the literature, submucosal invasion is associated with a relatively high risk of lymphatic metastasis since nearly one-third of the patients were identified as having positive lymph nodes during esophagectomy $(19,20)$. Several studies were designed to investigate predictors for nodal metastasis using pathologic factors in order to identify a low-risk subgroup of $\mathrm{T} 1 \mathrm{~b}$ cancer $(21,22)$. In accordance with previous studies $(5,23)$, ours demonstrated that tumor length, LVI $(0-30 \% \mathrm{~T} 1 \mathrm{~b}$ patients were reported to be have positive findings of LVI by previous studies) and invasion depth, were related to lymph node metastasis in submucosal ESCC. However, none of these pathologic factors alone was strong enough to identify a subgroup with an incidence of lymphatic metastasis lower than $20 \%$ as shown in our results. In this situation, it was obviously too risky to perform ER without resection of lymph nodes based on a single indicator.

The relationship between endoscopic morphology and frequency of nodal metastasis in superficial esophageal cancer has never been well studied. In the present study, flat lesions were found with a significantly lower nodal metastasis incidence $(15.5 \%)$, in comparison with nonflat type (35.9\%). In order to exclude other interference factors, multivariable analysis was performed, and the results implied an endoscopic shape of the tumors was an independent predictor for a lymphatic metastasis. Compared to other pathologic factors, endoscopic morphology allows surgeons to assess the lymph node status prior to a surgical resection, so that more suitable surgical treatment can be decided at an initial diagnosis. 
Our previous study has reported a logistic regression model using several pathologic factors for predicting the frequency of a lymphatic metastasis in T1b ESCC patients. Based on the model, a subgroup of T1b patients with the pathologic features of tumor length less than $2 \mathrm{~cm}$, well differentiation, tumor invasion of superficial submucosa and no LVI was found to have a lower risk of nodal metastasis (3). Some studies defined the "low risk" subgroup with similar pathologic features, and suggested that these selected submucosal esophageal carcinomas can be treated endoscopically $(7,24,25)$. However, the results were less than satisfactory, since the incidence of nodal metastasis was still above 10 percent even in the "low risk" subgroup. In the present study, the predictive model was improved by adding the endoscopic morphology procedure as an additional variate. What's more, instead of a mixed $\mathrm{T} 1 \mathrm{a} / \mathrm{T} 1 \mathrm{~b}$ group, our study population was focused on the pure patient population with submucosal carcinoma. Our study showed that if the endoscopic appearance is classified as flat type, and the other pathologic factors match the aforementioned "low risk" subgroup, the frequency of lymph node metastasis decreases to $7 \%$ according to the model. Thus, patients with this clinicopathological feature, especially those who are unfit for an esophagectomy or unwilling to receive it, might be suitable candidates for an endoscopic resection.

We acknowledged that this study has several limitations. First, it was a retrospective study preformed in a single center. Secondly, no survival analysis was performed in this study to assess the prognostic value of macroscopic morphology and the model, since the recent study reported the comparison of survival between $\mathrm{T} 1$ patients who received local therapy and those who underwent esophagectomy (26).

In conclusion, our results indicate that tumor in flat shape (0-II type) is related to less lymphatic metastasis, and assessing the endoscopic morphology along with other four pathologic variables can yield a more robust approach to predict the risk of lymphatic metastasis for submucosal ESCC.

\section{Acknowledgements}

Funding: This work was supported by the Shanghai Science and Technology Commission Foundation key project (14JC1401400), and a grant from the Science and Technology Commission of Shanghai Municipality (No.
15411951602; No. 16401970704).

\section{Footnote}

Conflicts of Interest: The authors have no conflicts of interest to declare.

Ethical Statement: This study was approved by Fudan University Shanghai Cancer Center Institutional Review Board (No. 090977-1). Informed consent was obtained from all individual participants included in the study.

\section{References}

1. Ishihara $\mathrm{R}$, Iishi $\mathrm{H}$, Uedo $\mathrm{N}$, et al. Comparison of EMR and endoscopic submucosal dissection for en bloc resection of early esophageal cancers in Japan. Gastrointest Endosc 2008;68:1066-72.

2. Shi Q, Zhong YS, Yao L, et al. Endoscopic submucosal dissection for treatment of esophageal submucosal tumors originating from the muscularis propria layer. Gastrointest Endosc 2011;74:1194-200.

3. Li B, Chen H, Xiang J, et al. Prevalence of lymph node metastases in superficial esophageal squamous cell carcinoma. J Thorac Cardiovasc Surg 2013;146:1198-203.

4. Gertler R, Stein HJ, Schuster T, et al. Prevalence and Topography of Lymph Node Metastases in Early Esophageal and Gastric Cancer. Ann Surg 2014;259:96-101.

5. Bolton WD, Hofstetter WL, Francis AM, et al. Impact of tumor length on long-term survival of p T1 esophageal adenocarcinoma. J Thorac Cardiovasc Surg 2009;138:831-6.

6. Ancona E, Rampado S, Cassaro M, et al. Prediction of lymph node status in superficial esophageal carcinoma. Ann Surg Oncol 2008;15:3278-88.

7. Higuchi K, Tanabe S, Koizumi W, et al. Expansion of the indications for endoscopic mucosal resection in patients with superficial esophageal carcinoma. Endoscopy 2007;39:36-40.

8. Bennett C, Vakil N, Bergman J, et al. Consensus statements for management of Barrett's dysplasia and early-stage esophageal adenocarcinoma, based on a Delphi process. Gastroenterology 2012;143:336-46.

9. Whiteman DC, Appleyard M, Bahin FF, et al. Australian clinical practice guidelines for the diagnosis and management of Barrett's esophagus and early esophageal adenocarcinoma. J Gastroenterol Hepatol 2015;30:804-20. 
10. Cummings LC, Kou TD, Schluchter MD, et al. Outcomes after endoscopic versus surgical therapy for early esophageal cancers in an older population. Gastrointest Endosc 2016;84:232-40.e1.

11. Qi ZP, Chen T, Li B, et al. Endoscopic submucosal dissection for early esophageal cancer in elderly patients with relative indications for endoscopic treatment. Endoscopy 2018;50:839-45.

12. The Paris endoscopic classification of superficial neoplastic lesions: esophagus, stomach, and colon: November 30 to December 1, 2002. Gastrointest Endosc 2003;58:S3-43.

13. Oda I, Abe S, Kusano C, et al. Correlation between endoscopic macroscopic type and invasion depth for early esophagogastric junction adenocarcinomas. Gastric Cancer 2011;14:22-7.

14. Lim MS, Lee H-W, Im H, et al. Predictable factors for lymph node metastasis in early gastric cancer-analysis of single institutional experience. J Gastrointest Surg 2011;15:1783-8.

15. Nagawa H, Kaizaki S, Seto Y, et al. The Relationship of Macroscopic Shape of Superficial Esophageal Carcinoma to Depth of Invasion and Regional Lymph Node Metastasis. Cancer 1995;75:1061-4.

16. Dutkowski P, Hommel G, Bottger T, et al. How many lymph nodes are needed for an accurate $\mathrm{pN}$ classification in esophageal cancer? Evidence for a new threshold value. Hepatogastroenterology 2002;49:176-80.

17. Shao Y, Ning Z, Chen J, et al. Prognostic nomogram integrated systemic inflammation score for patients with esophageal squamous cell carcinoma undergoing radical esophagectomy. Sci Rep 2015;5:18811.

18. Pech O, Bollschweiler E, Manner H, et al. Comparison between endoscopic and surgical resection of mucosal

Cite this article as: Zhuge L, Wang S, Xie J, Huang B, Zheng D, Zheng S, Mao H, Pennathur A, Sanchez MV, Luketich JD, Xiang J, Chen H, Zhang J. A model based on endoscopic morphology of submucosal esophageal squamous cell carcinoma for determining risk of metastasis on lymph nodes. J Thorac Dis 2018;10(12):6846-6853. doi: 10.21037/jtd.2018.11.77 esophageal adenocarcinoma in Barrett's esophagus at two high-volume centers. Ann Surg 2011;254:67-72.

19. Tachibana M, Hirahara N, Kinugasa S, et al. Clinicopathologic features of superficial esophageal cancer: results of consecutive 100 patients. Ann Surg Oncol 2008;15:104-16.

20. Grotenhuis BA, van Heijl M, Zehetner J, et al. Surgical management of submucosal esophageal cancer: extended or regional lymphadenectomy? Ann Surg 2010;252:823-30.

21. Davison JM, Landau MS, Luketich JD, et al. A Model Based on Pathologic Features of Superficial Esophageal Adenocarcinoma Complements Clinical Node Staging in Determining Risk of Metastasis to Lymph Nodes. Clin Gastroenterol Hepatol 2016;14:369-77.e3.

22. Qi X, Li M, Zhao S, et al. Prevalence of metastasis in T1b esophageal squamous cell carcinoma : a retrospective analysis of 258 Chinese patients. J Thorac Dis 2016;8:966-76.

23. Leers JM, DeMeester SR, Oezcelik A, et al. The prevalence of lymph node metastases in patients with T1 esophageal adenocarcinoma a retrospective review of esophagectomy specimens. Ann Surg 2011;253:271-8.

24. Endo M, Yoshino K, Kawano T, et al. Clinicopathologic analysis of lymph node metastasis in surgically resected superficial cancer of the thoracic esophagus. Dis Esophagus 2000;13:125-9.

25. Kim DU, Lee JH, Min BH, et al. Risk factors of lymph node metastasis in T1 esophageal squamous cell carcinoma. J Gastroenterol Hepatol 2008;23:619-25.

26. Kamel MK, Lee B, Rahouma M, et al. T1N0 oesophageal cancer: patterns of care and outcomes over 25 years. Eur J Cardiothorac Surg 2018;53:952-9. 

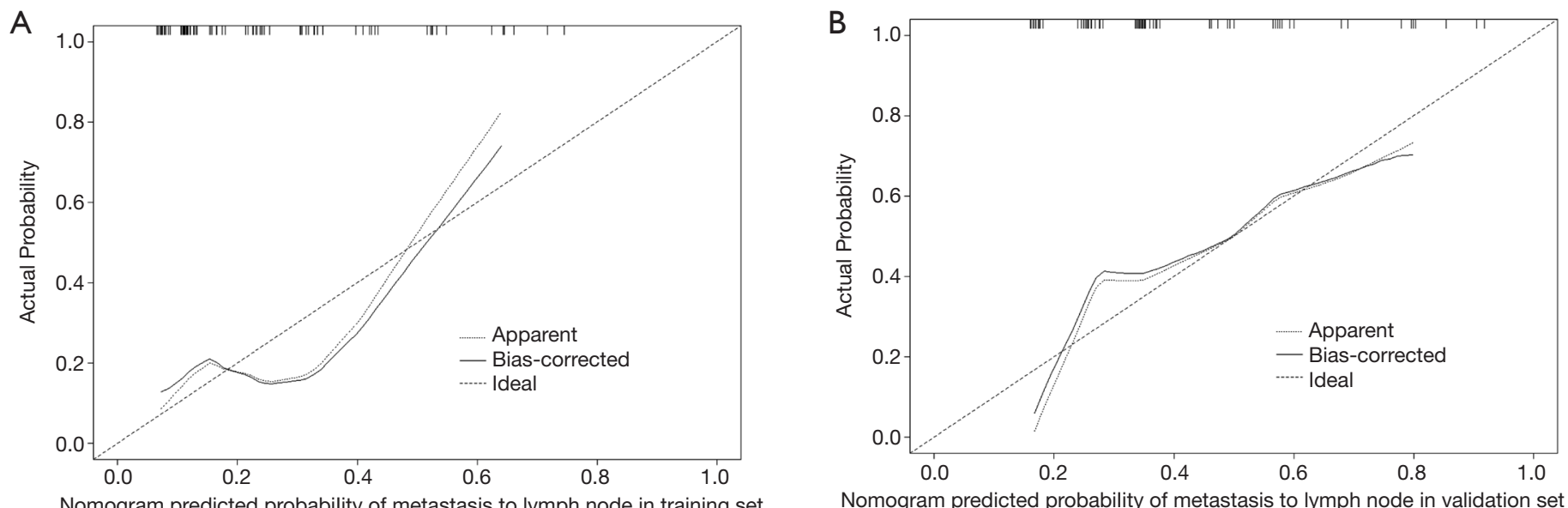

Figure S1 The calibration curve for predicting metastasis to lymph node in the training set (A) and validation set (B). 